\title{
A Type of Automatic Doffer for Conventional Ring Spinning Frame
}

\author{
By Aritsune Moriyama and Eiichi Yagita, Members, TMS
}

\author{
Daiwa Spinning Co., Ltd., Osaka
}

\begin{abstract}
This automatic cop doffer (A.C.D.) can be used directly on the conventional ring spinning frame with only very few modifications of the frame. Its major features are :

(1) The distance from the center line of the spindle to the outside of the A.C.D. is $320 \mathrm{~mm}$. The breadth of the carriage of A.C.D. is $945 \mathrm{~mm}$, which leaves enough floor space for the locomotion of the carriage on the other side.

(2) Use of a gravity shooting system for cop-arranging and feeding of bare bobbins simplifies mechanisms and conduces to accurate operations.

(3) A double elastic holder system being used to grip both cops and bobbins, lack of uniformity in the outer diameter of the bobbin does not affect doffing.

(4) Despite variations in the relative height of the spindle rail to the floor or floor rail, the body frame of A.C.D. can be held in the same fixed relative position to the spindle rails by adjusting the bottom wheel's fixed position.

(5) Use of friction wheels to drive A.C.D. along the frame side simplifies mechanisms and reduces operating costs. No rail connection from the carriage of A.C.D. is needed.
\end{abstract}

\section{Introduction}

Individual-doffing systems have been developed by pioneers of the postwar rebuilding of the Japanese spinning industry, including the Kanegafuchi Spinning Co., (Konebo), the Osaka Kiko Co. and the Itami Kogyo (formerly Kaneki). These systems have been followed by the later invention of groupdoffing sysiems, called the English type. Today we see various types of unique doffing systems in service. We are filled with admiration anew for ths work by the research staffs of the companies who have devoted themselves to bringing the various doffing systems to the high degree of perfection we see today.

It is trite to stress the need for Automatic Cop Doffer (A.C.D.), but it is not trite to stress the need for a type of A.C.D. system which can be installed in conventional mills not specially designed for the use of A.C.D. - a type mill magers really want. This article deals mainly with a type A.C.D. system which does group doffing and which can be installed with very few modifications of spinning rooms and spinning frames.

\section{Basic Idea and Merits of This Type}

To install A.C.D. in a conventional mill, the following three conditions must be filled:

(1) Use of the spindles and roller parts of the existing conventional spinning frame without changing their relative sizes and positions.

(2) Freedom from the need to change the beam height of the room. Freedom from the need to adjust the disparity between the frame level of the spinning frames and the ground.

(3) The width of A.C.D. should be as small as possible, because the clearance between the colums of the spinning room, on the one hand, and the frame, on the other, is usually limited.

Conditions 1 and 2 can be filled fairly easily. Condition 3 is apparently difficult to fill, because the minimum clearance in conventional mills is usually $200-250 \mathrm{~m} / \mathrm{m}$, theoretically too small for A.C.D. designed in the usual way to fit in.

To get around this problem, we initially conceived the idea of a system which would run only on one side of a frame, but do automatic doffing on both sides simultaneously by the pneumatic doffing of cops and pneumatic insertion of bobbins. Trial production of this system revealed some difficulty, however, the cop box and the bobbin box placed only on one side of the frame, and in spacing the pneumatic power sources. 
Admittedly, this difficulty would easily be overcome by using a pneumatic transportation system between processes, a conveyor system along the spinning frame side or a centralized pneumatic source system. However, installation expenses and high operating costs make the use of this solution prohibitive.

We then thought the width of A.C.D. system could be reduced considerably by using A.C.D. only for the transportation of bare bobbins and doffing and installing conveyor equipment on the sides of the frame to carry full cops one by one. This idea, however, was found inpracticable when we thought of the installation and maintenance costs of the conveyor equipment.

Finally we hit upon a type of A.C.D. which would contain a cop box inside which would have only a minimum width for simple mechanisms and operations. Here are the advantages of this type:

(1) Doffing motions are simulated to the conventional manual operations. This A.C.D. can be attached to conventional spinning frames.

(2) Despite variation in the relative height of the spindle rail to the floor or floor rail, the body frame of A.C.D. can be held in the same fixed relative position to the spindle rail by adjusting the bottom wheel's fixed position. The floor level need not be adjusted and none of the troubles usually caused by the construction of horizontal floor rails are met with.

(3) Use of friction wheels to drive A.C.D. along the frame side simplifies mechanisms and reduces costs. The carriage and the rails need not be connected. Moreover, even if there is a difference between the spindle rail level and the floor level where the carriage travels, this type of A.C.D. can be moved easily from the carriage of A.C.D. to the frame rails by separate adjustment of the end surface of the friction wheel rail attached to the frame side.

(4) Use of a gravity shooting system for cop-arranging and feeding of bare bobbins simplifies mechanisms and conduces to accurate operations.

(5) A double elastic holder system being used to grip cops and bobbins, want of uniformity in the outer diameter of the bobbin does not affect doffing.

\section{Main Mechanisms and Outline of the Operations}

Fig. 1 shows the outward look of this type.
Fig. 2 shows the layout dimensions. Here is the outline of the main mechanism and operation:

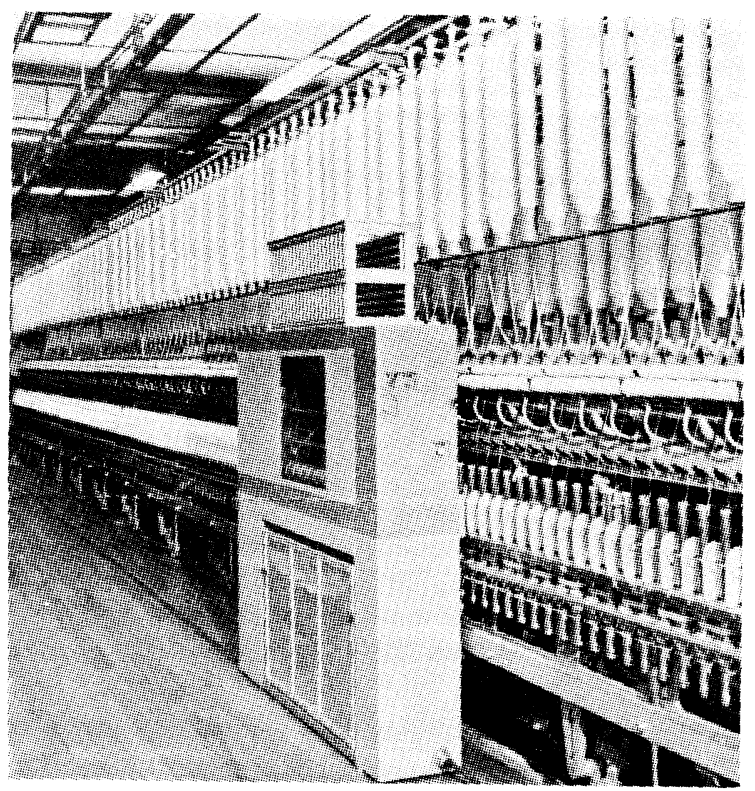

Fig. 1 Outward look of A.C.D.

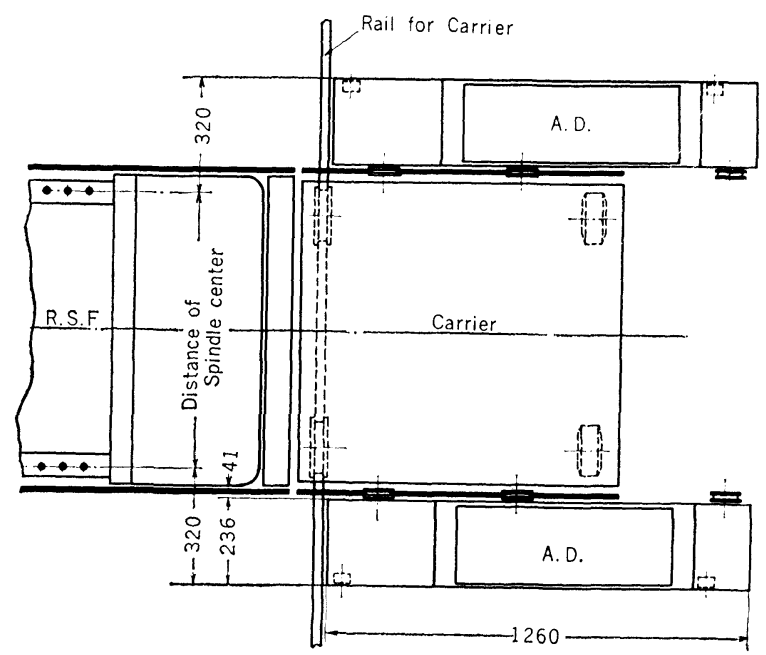

Fig. 2 Layout dimensions

\section{3-1. Cop Kicking up and Buffer Mechanism}

Fig. 3 shows the outline of the cop-kicking up mechanisms. The lever (1) revolves with the fulcrum (3) on the moving plate (2), the fulcrum being the center of the revolution. The plate (2) is driven back and forth by a cam. Another cam actuates lever (4), which, in turn, actuates lever (1). The lever (1) turns in two stages. Stage 1 loosens the insertion of cops. In stage 2, the lever (1) operates as a bobbin insertion buffer to prevent shockinduced yarn breakdowns. 

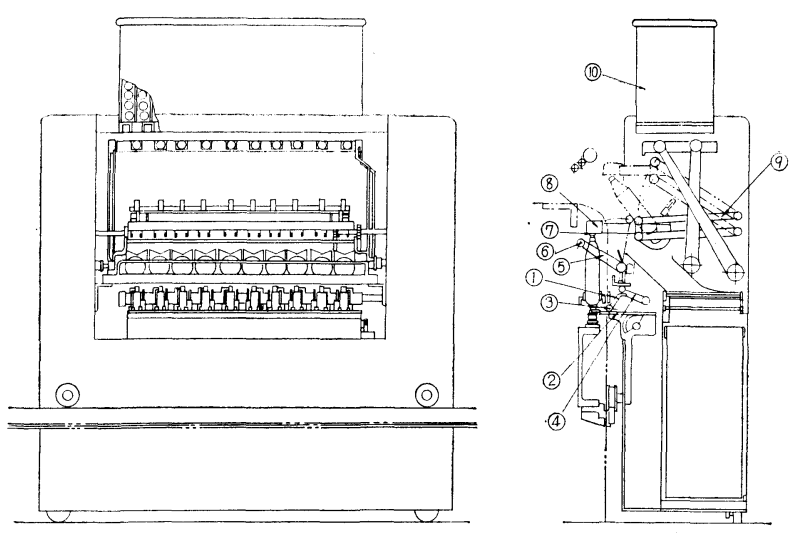

Fig. 3 Outline of A.C.D.

\section{3-2. Yarn and Cop Guide Mechanism}

A pair of levers (5) supports the rod (6) as shown in Fig. 3. Levers (5), turning the rod (6) through the operation of the cam, guides yarns toward the frame befo.e cop-doffing and guides the bottom ends of doffed cops in the direction of copshooting.

\section{3-3. Cop-doffing Mechanism}

All cop nippers (7) in Fig. 3 being springy operate together by the nipping force produced by the elastomer placed inside the head (8). In other words, the disparities in cop diameter between spindles in the group is adjusted by the elastic nipping force of the cop nippers (7). The cop is shifted, by the link motion (9), to the position shown by dotted lines in Fig. 3. During shifting, the cop makes a compound circular locus by the upward and downward movements of the link motion. After reaching the position just mentioned, the cop is separated from the head (8) by the above-mentioned guide.

\section{3-4. Severing and Holding of Yarn End}

A rough sketch of this mechanism is given in Fig. 4. The end yarn stretching from the spindle (1) to the full wound bobbin (2) is conveyed to the slits in guides (3) and (4) and severed by a highspeed cutter (5) installed between the two guides. The end yarn is then sucked into the suction hole (6) and held mildly in it until an empty bobbin is impaled on the spindle.

\section{3-5. Cop-arranging Mechanism}

Fig. 5 shows the interior of this mechanism. Doffed cops drop from the above-mentioned doffing mechanism to the receiving plate (1) which is horizontally placed. The cops are dropped between the sheets (3) and $\left(3^{\prime}\right)$ as the plate (1) is rotated by the operation of the solenoid (2). The bottom ends of the sheets contain a pair of magnets (4) and $\left(4^{\prime}\right)$ which differ in polarity. Falling cops are re-

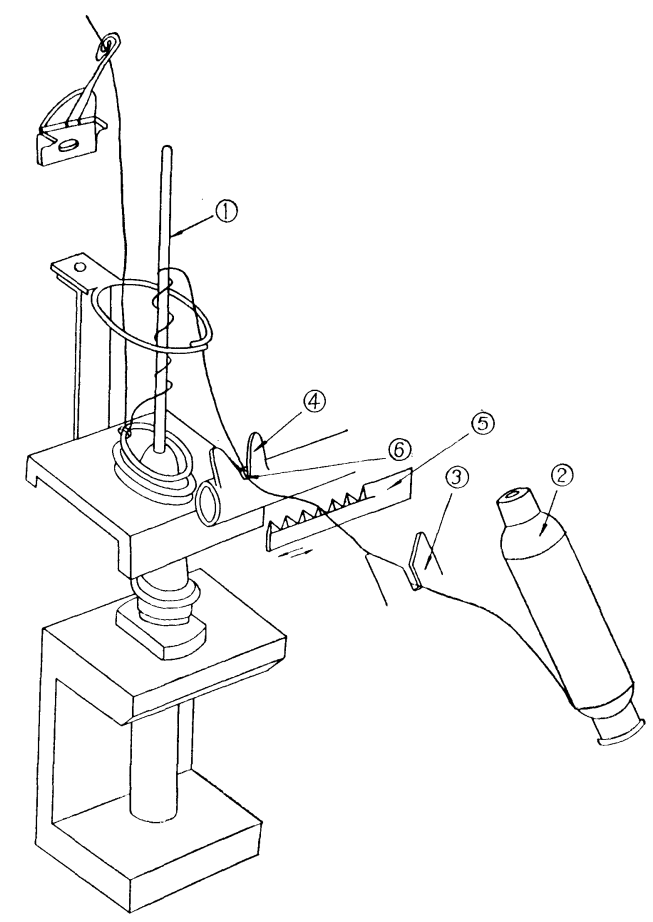

Fig. 4 Severing and holding of end yarn

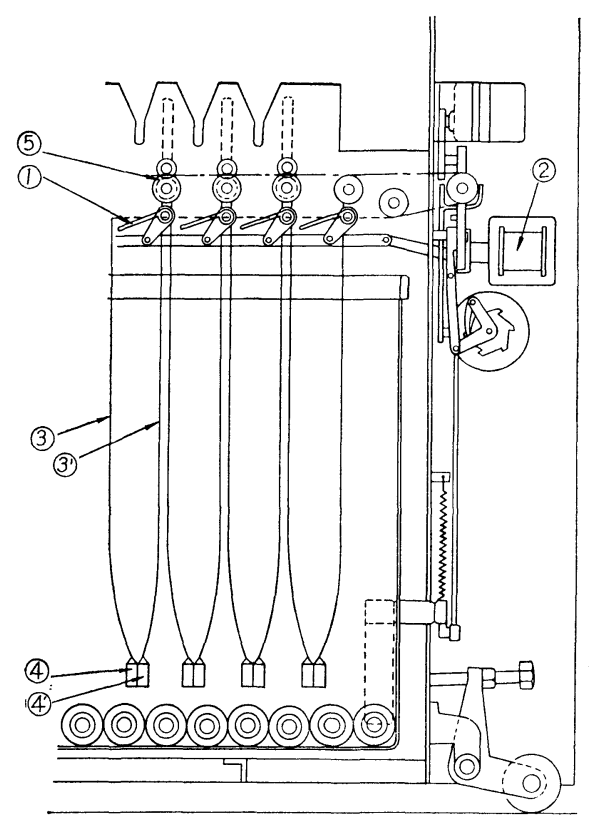

Fig. 5 Cop-arranging mechanisms 
ceived by the sheets, keeping their horizontal posture, and stacked in the bottom of the box. Sheets (3) and (3') are gradually wound by a pair of rollers (5) while cops are stacked to fill the box.

\section{3-6. Bobbin Insertion}

The bobbin box is installed on the top of A.C.D., as shown in Fig. $3(10)$. Fig. 6 shows the interior of the box. Bobbins are fed one by one to bobbin receivers (2) as the box-type damper (1) is driven by the cam.

Fig. 7 is a sketch of the bare bobbin insertion mechanisms. Cross-link mechanism (3) and $\left(3^{\prime}\right)$ are driven by the cam (1) and cam lever (2). The bobbin receiver (2) shown in Fig. 6, which contains a double elastic holder system like the one mentioned before, changes its position quickly from horizontal to vertical for descent toward the position of the spindles.

\section{3-7. Travelling Mechanism}

A rough sketch of this system is given in Fig. 8. Starting and stopping of the driving wheel (2), which travels on the guide rails (1), is governed by the movable clutch (3). The fork (5) moving with A.C.D. is turned from position (A) to position (B) by the position-setting pin (4) fixed on the rail (1) with a pitch appropriate to the distance between spindles in the group. The turning of this fork (5) is stopped by moving off the clutch (3) through the operation of the wheel (6), thus putting the magnetic brake (7) to work and causing the knock pin (8) to come to the hole of the wheel (6) and govern the prescribed stop position.

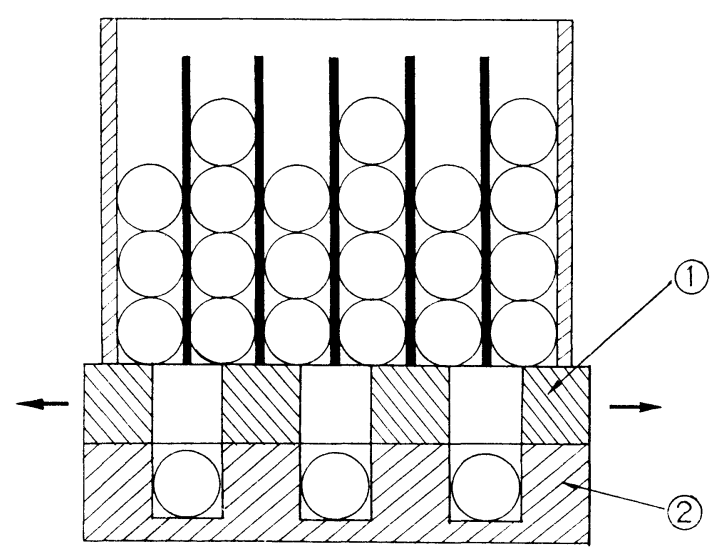

Fig. 6 Interior of bare bobbin box

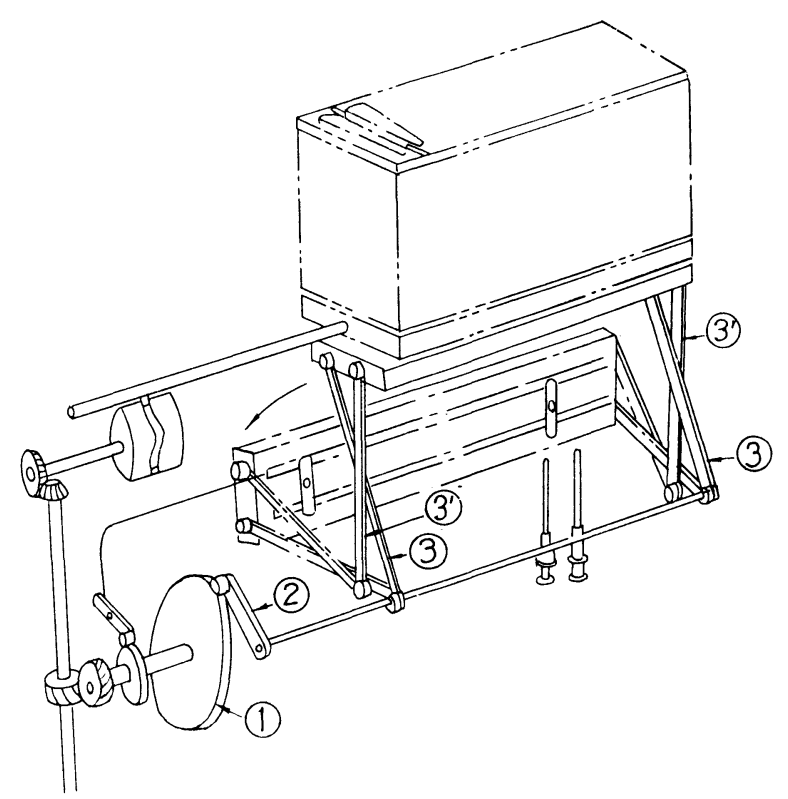

Fig. 7 Bare-bobbin inserting mechanisms

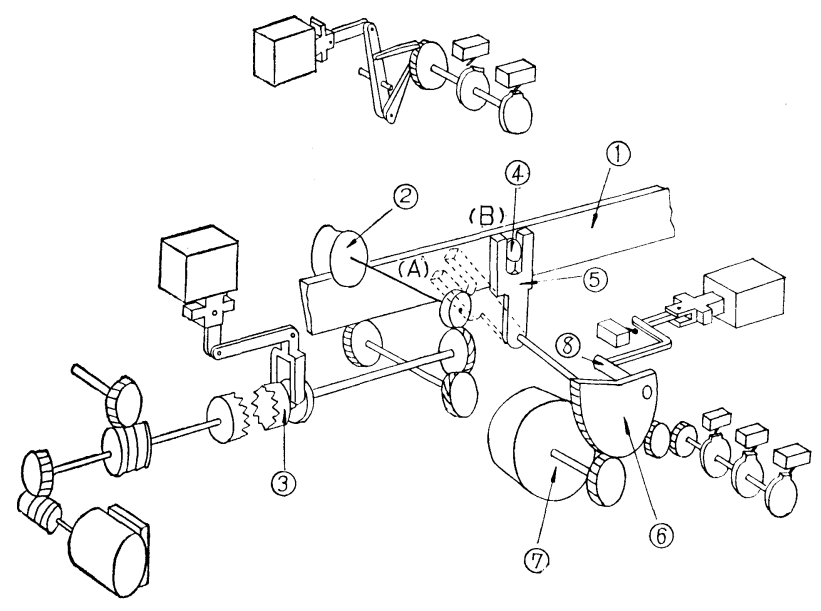

Fig. 8 Travelling mechanisms

\section{Postscript}

A new A.C.D. system which is still in the blueprint stage will be reported upon at a future date. As we said in their article, many conditions have to be filled for the use of the A.C.D. system. We hope the time will come before long when the A.C.D. systems of all types can be put into service in conventional mills. The authors owe a debt of gratitude to the Daiwa Spinning Company for their generous supply of funds and labor for the developement of A.C.D. 